\title{
Monocarboxylate Transporter 2 and Stroke Severity in a Rodent Model of Sleep Apnea
}

\author{
Yang Wang, ${ }^{1}$ Shang Z. Guo, ${ }^{2}$ Arend Bonen, ${ }^{3}$ Richard C. Li, ${ }^{1}$ Leila Kheirandish-Gozal, ${ }^{1}$ Shelley X. L. Zhang, \\ Kenneth R. Brittian, ${ }^{2}$ and David Gozal ${ }^{1}$ \\ ${ }^{1}$ Department of Pediatrics, University of Chicago, Chicago, Illinois 60637, 2Department of Pediatrics, University of Louisville School of Medicine, Louisville, \\ Kentucky 40202, and ${ }^{3}$ Department of Human Health and Nutritional Sciences, University of Guelph, Guelph, Ontario, Canada N1G 2W1
}

Stroke is not only more prevalent but is also associated with more severe adverse functional outcomes among patients with sleep apnea. Monocarboxylate transporters (MCT) are important regulators of cellular bioenergetics, have been implicated in brain susceptibility to acute severe hypoxia (ASH), and could underlie the unfavorable prognosis of cerebrovascular accidents in sleep apnea patients. Rodents were exposed to either intermittent hypoxia $(\mathrm{IH})$ during sleep, a characteristic feature of sleep apnea, or to sustained hypoxia (SH), and expression of MCT1 and MCT2 was assessed. In addition, the functional recovery to middle cerebral artery occlusion (MCA0) in rats and hMCT2 transgenic mice and of hippocampal slices subjected to ASH was assessed, as well as the effects of MCT blocker and MCT2 antisense oligonucleotides and siRNAs. IH, but not SH, induced significant reductions in MCT2 expression over time at both the mRNA and protein levels and in the functional recovery of hippocampal slices subjected to ASH. Similarly, MCAO-induced infarcts were significantly greater in IH-exposed rats and mice, and overexpression of hMCT2 in mice markedly attenuated the adverse effects of IH. Exogenous pyruvate treatment reduced infarct volumes in normoxic rats but not in IH-exposed rats. Administration of the MCT2 blocker $4 \mathrm{CN}$, but not the MCT1 antagonist $p$-chloromercuribenzene sulfonate, increased infarct size. Thus, prolonged exposures to IH mimicking sleep apnea are associated with increased CNS vulnerability to ischemia that is mediated, at least in part, by concomitant decreases in the expression and function of MCT2. Efforts to develop agonists of MCT2 should provide opportunities to ameliorate the overall outcome of stroke.

\section{Introduction}

Obstructive sleep apnea (OSA), which affects $4-6 \%$ of the adult middle-age population, is characterized by repeated episodes of hypoxia and hypercapnia during sleep as well as with sleep discontinuity attributable to frequent arousals aiming to reestablish upper airway patency. The major deleterious consequences of untreated OSA can be partitioned into two major groups, namely cardiovascular and neural morbidities. From the cardiovascular standpoint, OSA, via repeated arousals and/or intermittent hypoxia (IH), may play a causative role in hypertension, which in turn could accelerate the onset or aggravate the clinical course of cardiovascular disease and increase the risk for sudden death (Somers et al., 2008). The major neurocognitive manifestations of OSA include excessive daytime sleepiness, personality and psy-

\footnotetext{
Received March 22, 2011; revised May 12, 2011; accepted May 18, 2011.

Author contributions: Y.W. and D.G. designed research; Y.W., S.Z.G., A.B., R.C.L., L.K.-G., S.X.L.Z., K.R.B., and D.G. performed research; D.G. analyzed data; Y.W. and D.G. wrote the paper.

The authors declare no competing financial interests.

This study was supported by National Institutes of Health (NIH) Grant HL-086662 and NIH Specialized Centers of Research Grant 2P50HL-60296 (D.G.), American Heart Association Grant AHA-0930129N (R.C.L.), and the Natural Sciences and Engineering Research Council of Canada and the Canada Research Chair Program (A.B.). We are grateful to Drs. Luc Pelerin and Pierre Magistretti for graciously providing access to some of the MCT antibodies used in this study.

Correspondence should be addressed to David Gozal, Department of Pediatrics, Pritzker School of Medicine, Comer Children's Hospital, The University of Chicago, 5721 South Maryland Avenue, MC 8000, Suite K-160, Chicago, IL 60637. E-mail: dgozal@uchicago.edu.

DOI:10.1523/JNEUROSCI.1462-11.2011

Copyright $\odot 2011$ the authors $\quad 0270-6474 / 11 / 3110241-08 \$ 15.00 / 0$
}

chosocial maladjustment patterns, and mental impairment in terms of thinking, perception, memory, communication, or the ability to learn new information (Beebe et al., 2003; Kheirandish and Gozal, 2006; Nowak et al., 2006). Similarly, an increased prevalence of stroke among OSA patients has been repeatedly reported (Sahlin et al., 2008; Valham et al., 2008), whereby OSA is a risk factor for stroke as well as a consequence of stroke (Minoguchi et al., 2007; Ali and Avidan, 2008). Furthermore, the severity and anatomical extent of the stroke appears to be exacerbated in the presence of OSA (Parra et al., 2000), with both the shortterm and long-term outcomes of stroke being worse in patients suffering from OSA (Spriggs et al., 1992; Good et al., 1996; Bassetti and Aldrich, 1999; Ryan et al., 2011).

Sustained hypoxia $(\mathrm{SH})$ confers neural protection in a rodent model of acute hypoxia-ischemia, possibly via a coordinated regulation of multiple gene networks (Ratan et al., 2007). However, these findings apparently contradict those of increased stroke severity among patients with OSA. These disparities suggest that $\mathrm{IH}$ and $\mathrm{SH}$ may greatly differ in their effects on gene regulation and on their ability to mount protective neural survival mechanisms after acute ischemia. The mechanisms mediating the increased brain vulnerability to acute ischemia when IH is present during sleep are unknown.

Lactate accumulates in the brain not only during oxygen deprivation but also during normal cerebral stimulation in normoxic conditions (Fellows et al., 1993). Moreover, lactate can support synaptic function in vitro in the absence of glucose as the 
sole energy substrate (Schurr et al., 1988). In acute severe hypoxia (ASH), glycolysis becomes essential for ATP production, and the concentration of monocarboxylates significantly rises in neural tissue (Schurr et al., 1997a). The protective role of such lactate increase during ASH is further supported by the observations that lactate is preferred over glucose and is an obligatory energy substrate during functional neural recovery after ASH (Schurr et al., 1997b). The coupling of glutamatergic neuronal activity in the brain and glucose and subsequent lactate utilization is explained by the existence of an astrocyte-neuron lactate shuttle (Pellerin and Magistretti, 1994). Under such mechanism, lactate is released from astrocytes via monocarboxylate transporter 1 (MCT1) and undergoes uptake by neurons through MCT2, in which it is used to fulfill the energetic needs of these cells. If lactate transport is impaired, lactic acid will accumulate, resulting in a decrease in $\mathrm{pH}$, an inhibition of glycolysis, a decrease in cellular ATP, and ultimately leading to cell death in the astrocytes and to energy deprivation and cell dysfunction or death in neurons (Schurr and Rigor, 1998; Berthet et al., 2009; Allaman et al., 2011; Suzuki et al., 2011). Thus, the increased neural tissue vulnerability to ASH in OSA could result from maladaptive changes in the expression of lactate transporters MCT1 and MCT2, leading to an imbalance between lactate utilization and transport and compromising the ability of neurons to uptake monocarboxylates as obligatory energy substrates for functional recovery from ASH.

\section{Materials and Methods}

\section{Animals}

Male Sprague Dawley rats (200-225 g; Charles River) and C57BL/6 (The Jackson Laboratory) and transgenic mice overexpressing the human MCT2 (hMCT2 Tg) were used for experiments. The experimental protocols were approved by the Institutional Animal Use and Care Committee and are in close agreement with the National Institutes of Health Guide for the Care and Use of Laboratory Animals. All efforts were made to minimize animal suffering, to reduce the number of animals used, and to use alternatives to in vivo techniques. hMCT2 $\mathrm{Tg}$ mice were generated after cloning the open-reading frame of hMCT2 using a PCR-based strategy and subsequently generating a ubiquitin $\mathrm{C}$ promoter-based hMCT2 overexpression vector that also possessed a 3' V5-His tag for easy detection of the fusion protein. DNA injections were performed using eggs derived from a C57BL/ $6 \times \mathrm{DBA} 2(50 \times 50 \%)$ hybrid strain, followed by breeding the hMCT2 founders to identify nonchimeric, germ-linetransmitting F1 mice and backcrossing them with the C57BL/6 strain for at least 10 generations.

\section{Intermittent or sustained hypoxia exposures}

Animals sojourned in commercially designed chambers (Oxycycler model A44XO; Reming Bioinstruments) that were operated under a $12 \mathrm{~h}$ light/dark cycle (6:00 A.M. to 6:00 P.M.). Gas was circulated around each of the chambers, attached tubing, and other units at $60 \mathrm{~L} / \mathrm{min}$ (i.e., one complete change per 10-30 s). The $\mathrm{O}_{2}$ concentration was continuously measured by an $\mathrm{O}_{2}$ analyzer and was changed throughout daylight hours by a computerized system controlling the gas valve outlets, such that the moment-to-moment desired oxygen concentration of the chamber was programmed and adjusted automatically. Deviations from the desired concentration were met by addition of $\mathrm{N}_{2}$ or $\mathrm{O}_{2}$ through solenoid valves. For the remaining $12 \mathrm{~h}$ of nighttime, oxygen concentrations were kept at $21 \%$. Ambient $\mathrm{CO}_{2}$ in the chamber was periodically monitored and maintained at $<0.01 \%$ by adjusting overall chamber basal ventilation. Humidity was measured and maintained at $40-50 \%$ by circulating the gas through a freezer and silica gel. Ambient temperature was kept at $24^{\circ} \mathrm{C}$ for rats and $28^{\circ} \mathrm{C}$ for mice. The $\mathrm{IH}$ profile for rats consisted of alternating room air (RA) and 10\% oxygen every $90 \mathrm{~s}$ during daylight hours for the desired duration (Gozal et al., 2001), whereas SH consisted of constant exposure to $10 \%$ oxygen. For mice, a similar cycle duration was used, but $\mathrm{F}_{\mathrm{I}} \mathrm{O}_{2}$ nadir was $5.7 \%$. The frequency and duration of the $\mathrm{IH}$ cycles was aimed to reproduce the oxyhemoglobin desaturation patterns frequently found in patients with OSA or moderate severity. Time-pointmatched control animals were exposed to circulating normoxic gas in one of the four chambers.

\section{Preparation of hippocampal slices}

Animals were anesthetized with ether and perfused through the heart with $60 \mathrm{ml}$ of cold $\left(4^{\circ} \mathrm{C}\right)$ bathing medium containing the following (in $\mathrm{mm}): 129 \mathrm{NaCl}, 3.5 \mathrm{KCl}, 2 \mathrm{MgSO}_{4} 1 \mathrm{NaH}_{2} \mathrm{PO}_{4}, 2.7 \mathrm{CaCl} 2,26 \mathrm{NaHCO}_{3}$, and 10 glucose (Kreisman et al., 2000). The rats were decapitated, the brain was removed, and the tissue was placed immediately in ice-cold bathing medium. Transverse slices (300- $\mu \mathrm{m}$-thick) were cut with a mechanical tissue chopper (Stoelting) from the middle one-third of the hippocampus to avoid septotemporal gradients of excitability. Slices were then placed in the wells of a holding chamber on filter paper thoroughly wetted with bathing medium and gassed with humidified $95 \%$ $\mathrm{O}_{2}-5 \% \mathrm{CO}_{2}$. The incubation medium was maintained at room temperature $\left(23-24^{\circ} \mathrm{C}\right)$ and was replaced with fresh medium at $45 \mathrm{~min}$ intervals. After 90-120 min of preincubation, slices were transferred two each time to the nylon mesh of superfused-style recording chambers. The temperature in the recording chamber was maintained at $33-34^{\circ} \mathrm{C}$, and the slice was superfused with standard bathing medium flowing at $0.6 \mathrm{ml} / \mathrm{min}$. Warmed, humidified $95 \% \mathrm{O}_{2}-5 \% \mathrm{CO}_{2}$ superfused the slice at a rate of $480 \mathrm{ml} / \mathrm{min}$. Osmolarity of the standard bathing medium was 295-300 $\mathrm{mOsm} / \mathrm{L}$.

\section{Electrical field stimulation and recording}

Viability of the CA1 region was tested by stimulating the Schaffer collaterals with constant-current pulses ( $400 \mathrm{~mA}, 0.2 \mathrm{~ms}$ ) using glass micropipettes filled with $150 \mathrm{~mm} \mathrm{NaCl}$ (tip resistance, 5-20 mV). Only stable recordings of population spikes with a minimum amplitude of $3 \mathrm{mV}$ were acceptable. fEPSPs were recorded extracellularly in CA1 stratum radiatum in response to stimulating the Schaffer collaterals with constant-current pulses $(50-100 \mathrm{~mA}, 1 \mathrm{~ms})$ to produce a response that was $50-75 \%$ of maximum amplitude. Extracellular direct current levels were recorded continuously on a strip-chart recorder, and evoked responses were digitally acquired.

\section{Induction of in vitro hypoxia}

ASH was induced by switching the gas mixture superfusing the slices from $95 \% \mathrm{O}_{2}-5 \% \mathrm{CO}_{2}$ to $95 \% \mathrm{~N}_{2}-5 \% \mathrm{CO}_{2}$. In several experiments, $\mathrm{PO}_{2}$ in the bathing medium was measured polarographically, by using a platinum electrode polarized to $20.7 \mathrm{~V}$ relative to an $\mathrm{Ag}-\mathrm{AgCl}$ wire connected to ground. The mean $\mathrm{PO}_{2}$ values in the upper $1 \mathrm{~mm}$ of the bath during normoxia and hypoxia were $443 \pm 19$ and $20 \pm 3 \mathrm{mmHg}$, respectively $(n=12)$. The duration of hypoxia was restricted to 10 or $12 \mathrm{~min}$, followed by reoxygenation. Functional recovery was assessed by reappearance of fEPSP within $30 \mathrm{~min}$, provided that the amplitude of fEPSP was $>40 \%$ from baseline. In preliminary experiments, $\sim 85 \%$ of hippocampal slices derived from control rats displayed functional recovery after being exposed to $10 \mathrm{~min} \mathrm{ASH}$, whereas only $55 \%$ recovered after $12 \mathrm{~min}$. Therefore, a $10 \mathrm{~min}$ ASH duration was retained for all subsequent experiments.

\section{Middle cerebral artery occlusion}

(1) In rats under general anesthesia, a craniectomy was performed, and surgical exposure of the right MCA was conducted under direct visualization using surgical microscope. The artery was occluded using a surgical string at its entrance into the lateral base of the skull for $65 \mathrm{~min}$. Reperfusion was then allowed by releasing the occlusion, and reestablishment of blood flow to preligation levels was allowed and verified using laser Doppler flowmetry (Periflux 5000 System; Perimed). After 72 h, animals were killed, and the brain was extracted and stained using 2,3,5triphenyltetrazolium chloride or green methionine stain. (2) In another subset of rats, recovery was allowed for $14 \mathrm{~d}$, after which a water maze protocol was conducted, and the animals were killed and processed as above. Infarct volume was determined from images obtained from serial sections of perfused brains stained with vital stain and examined by a blinded investigator. For controls, a craniectomy was conducted, and the dura were opened for vessel exposure but no occlusion was done (sham). (3) In a third subset of rats, MCAO was conducted, and within $1 \mathrm{~h}$ from 


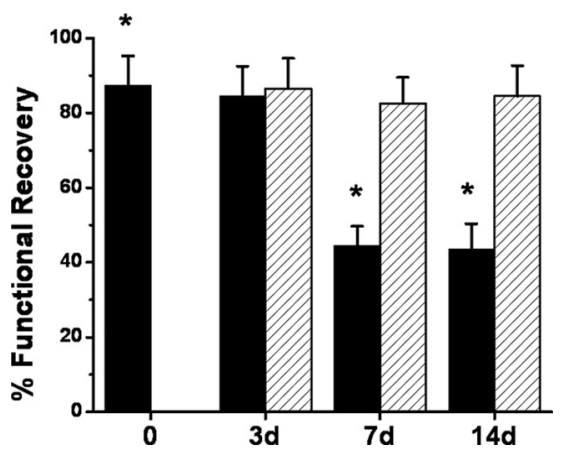

Figure 1. Hippocampal slices were exposed to ASH for $10 \mathrm{~min}$ after which reemergence of fEPSPs was assessed. No differences in ASH susceptibility emerged in SH-exposed rats after 3, 7, or $14 \mathrm{~d}$ exposures (red columns; $n=24$ slices from 6 different rats per group) when compared with RA-exposed rats (black column at time $0 ; n=48$ slices from 12 different rats per group). In contrast, although no changes occurred after $3 \mathrm{~d}$ of $\mathrm{IH}$, the percentage of slices harvested from rats exposed to either 7 or $14 \mathrm{~d} \mathrm{IH}$ showing functional recovery was reduced $\left({ }^{*} p<0.0001\right.$ vs time $0 ; n=24$ slices from 6 different rats per group).

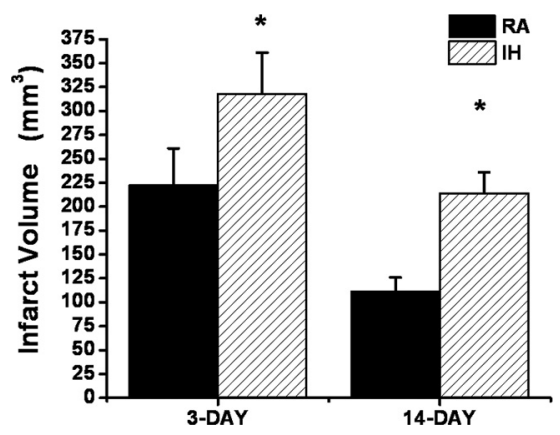

Figure 2. Infarct size after MCAO in adult rats after either 3 or $14 \mathrm{~d}$ ( $n=12 /$ group). Infarct volume was significantly larger at both time points after IH exposures when compared with normoxic controls (RA; $\left.{ }^{*} p<0.001\right)$.

reperfusion, either pyruvate $(500 \mathrm{mg} / \mathrm{kg}$ ) or vehicle was given intraperitoneally. Animals were allowed to recover for $3 \mathrm{~d}$, after which they were killed and processed as above for assessment of infarct size. (4) In a fourth group, anesthetized animals underwent placement of a cannula in the left lateral ventricle attached to an osmotic pump (Alzet model 1002 and brain infusion kit) filled with the MCT blocker cyano-4-hydroxycinnamate (4-CN) (2 mM; Sigma), the MCT1 blocker $p$-chloromercuribenzene sulfonate (pCMBS) ( $2 \mathrm{~mm}$; Sigma), or vehicle. In addition, $10 \mu \mathrm{l}$ of $2 \mathrm{~mm} 4-\mathrm{CN}, \mathrm{pCMBS}$, or vehicle were given intracerebroventricularly at the end of surgery. The concentration of $4-\mathrm{CN}$ was established from preliminary experiments designed to achieve an effective concentration of the compound within the $0.5 \mathrm{~mm}$ range in vitro (Schurr et al., 2001). In addition, this concentration has been established as preferentially blocking the activity of MCT2. Two days after recovery, MCAO was conducted as described above, and infarct size was determined after 3 d. (5) An antisense oligonucleotide (ON) specifically targeted against MCT2 and validated in preliminary in vitro experiments and scrambled oligonucleotide were administered intracerebroventricularly using an osmotic pump for $72 \mathrm{~h}$, followed by MCAO. (6) Finally, chemically synthesized siRNAs $(500 \mu \mathrm{g}$ in $100 \mu \mathrm{l})$ were initially injected into the left lateral ventricle in $10 \mathrm{~min}$ via the implanted cannula. This initial dose was followed by an intracerebroventricular infusion of the same siRNAs at a rate of $2.5 \mu \mathrm{g} / \mathrm{h}$ with the use of the osmotic pump for $5 \mathrm{~d}$. Three days after the initial dose, MCAO was conducted as above, and, $2 \mathrm{~d}$ later, infarct size was determined. Two siRNAs specific for rat MCT2 were shown to be effective in silencing the gene in cultured cells and were used in combination (1:1) for intracerebroventricular applications (rMCT2/1066, 5' AAGTAAGGTTGGCTCAAGACA-3'; rMCT2/1360, 5'AATCCGTCCACGAATCCAGTA-3').
A
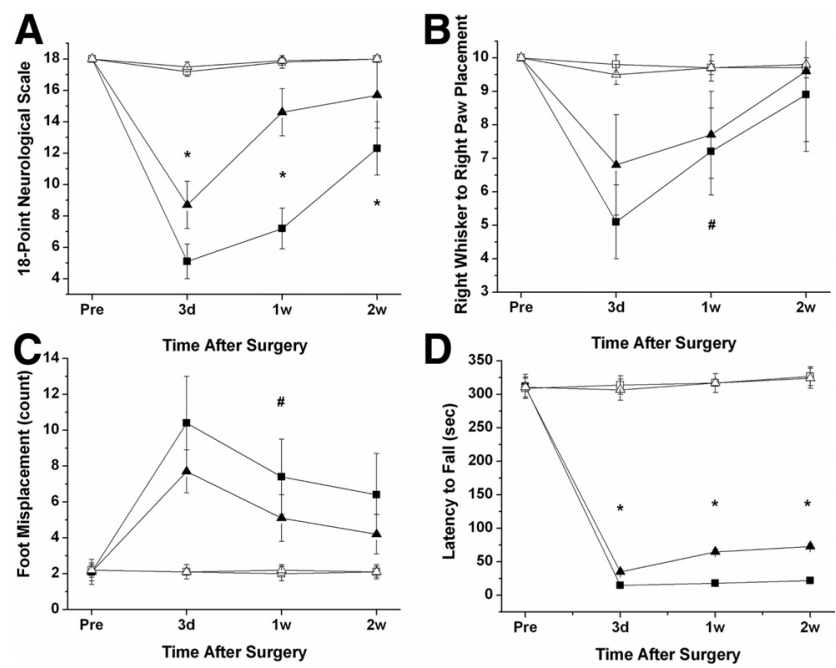

Figure 3. Behavioral test performances in rats subjected to MCAO (filled symbols) or sham (open symbols) after IH (square symbols) or RA (normoxic controls; triangle symbols). $\boldsymbol{A}$, Eighteen-point neurological scores were significantly lower in IH rats subjected to $\mathrm{MCAO}\left({ }^{*} p<\right.$ 0.01 , IH vs RA; $n=12$ ). $\boldsymbol{B}$, Whisker stimulation and paw placement test showed decreased responses in IH-exposed rats after MCAO compared with RA controls ( ${ }^{\#} p<0.04$, ANOVA). $\boldsymbol{C}$, Foot fault test performance tests showed increased number of misplacements in $\mathrm{IH}$-exposed rats after MCAO ( $\left.{ }^{\#} p<0.04, A N O V A\right)$. D , Rotarod test performances showed that rats subjected to IH exposures and MCAO had significantly shorter latencies than normoxic controls ${ }^{*} p<0.01$ vs RA).

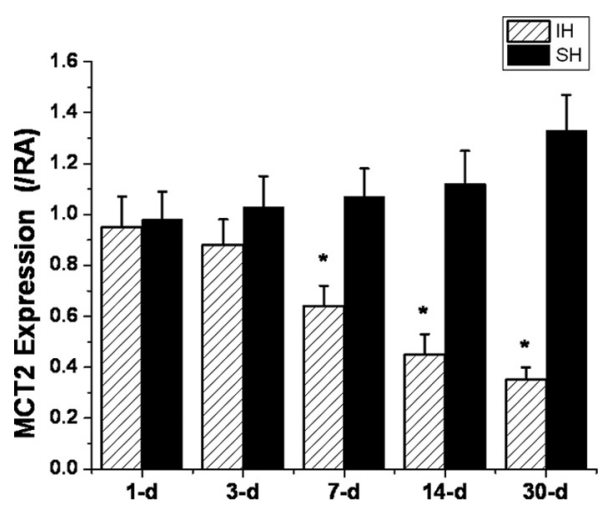

Figure 4. Quantitative RT-PCR for MCT2 in rat cortical lysates harvested from animals exposed to SH (black columns) or IH (red columns) for 1-30 d. Significant downregulation of MCT2 mRNA expression emerged at $7 \mathrm{~d}$ and continued to progress until $30 \mathrm{~d}$ of $\mathrm{IH}$; however, no significant changes in MCT2 expression occurred in SH-exposed animals $\left({ }^{*} p<0.01 ; n=\right.$ $6 /$ time point). All values are reported as ratios between condition ( $\mathrm{SH}$ or $\mathrm{IH}$ ) and time-matched room air controls.

\section{Antisense oligonucleotide approach}

A MCT2 knockdown strategy using phosphorothioate antisense ONs was developed to complement pharmacological inhibition of MCT2. We identified a $14 \mathrm{nt}$ sequence ( $5^{\prime}$-TGGCATTTCTGAGC-3') surrounding the translation initiation site of the rat MCT2 gene and found that this region shared relatively low degree of homology with other members of the MCT gene family. For example, it is 59 and $43 \%$ homologous with the rat MCT1 and MCT4 genes, respectively. The specificity and effectiveness of this antisense ON in inhibiting MCT2 protein synthesis were confirmed in a transient transfection assay in PC12 cells that constitutively express MCT2 and further verified in vivo before MCAO experiments. A scrambled ON (5'-CTGAGTGTCATGCT-3') was used as control.

Transient middle cerebral artery occlusion in mouse

hMCT2 Tg and wild-type mice (10-week-old) were anesthetized by an intraperitoneal injection of $8 \mathrm{mg} / \mathrm{kg}$ xylazine and $100 \mathrm{mg} / \mathrm{kg}$ ketamine. 
Ischemia was induced by inserting an $11 \mathrm{~mm}$ silicone-coated 8-0 filament through the left common carotid artery into the internal carotid artery. The filament was withdrawn after $30 \mathrm{~min}$, allowing reperfusion. Regional cerebral blood flow was measured by laser Doppler flowmetry (Periflux 5000; Perimed) with a flexible probe fixed on the skull, $1 \mathrm{~mm}$ posteriorly and $6 \mathrm{~mm}$ laterally from bregma. The mice were killed $48 \mathrm{~h}$ after the onset of focal ischemia, and $20-\mu \mathrm{m}$-thick, $720-\mu \mathrm{m}$-apart, coronal cryostat sections were stained with cresyl violet for histologic determination of lesion size. A digitalized image of the Nissl-stained tissue was obtained under a light stereomicroscope, and the lesion area on stained sections was determined by an examiner blinded for the treatment group using NIH ImageJ software (http://rsb.info.nih.gov/ij/). Direct infarct volume was calculated by multiplying the sum of the infarct areas on each section by the spacing distance. To avoid bias attributable to edema, an indirect lesion size was calculated as follows: Indirect lesion $=$ volume $_{\text {contralateral }}-\left(\right.$ volume $_{\text {ipsilateral }}-$ direct infarct volume) as described previously by Swanson et al. (1990).

\section{Behavioral tests}

Eighteen-point composite neurological score. The composite neuroscore comprises six different neurological tests: (1) spontaneous activity, (2) symmetry in limb movement, (3) forepaw outstretching, (4) climbing, (5) body proprioception, and (6) response to vibrissae touch. Each test is scored with a maximum of three points based on a set of predetermined criteria as described previously (Garcia et al., 1995). The scores for each test were summed for a highest possible score of 18 , indicating no neurological deficit, and the lowest score of 3 , for animals with the most severe impairment.

Foot fault test. The foot fault (Columbus Instruments) is used to measure the coordination of an animal walking across a horizontal ladder (Colle et al., 1986). The ladder width is 6.5 inches, with 0.3125 -inch beams 1 inch apart. The animal is trained to walk across a ladder 0.25 inches above a steel plate. The plate is then charged once during training so that, if the animal's paw comes in contact with the plate, the animal is lightly shocked. The animal learns consequently to avoid the steel plate by keeping its paws on the ladder beams during each run. Foot misplacements during the run and time to complete each run are counted. The average performance of three consecutive runs is reported for each animal.

Vibrissae-evoked forelimb placing. The forelimb placing test assesses the ability of rats to sense tactile stimulus from the vibrissae and subsequently elicit a motor response of forelimb placing (Schallert et al., 2003). In this test, animal body was held gently such that the paws were suspended off the experimenter's hand. The animal was then brought laterally to a table top while the vibrissae brush against the edge of the table, allowing the animal to reach the edge with its forepaw. This procedure was repeated 10 times on each side. The number of successful paw placements on to the edge of the table was recorded. A cross-midline variation to the test was also performed. Here, the animal was held on its side, and successful placements of the opposite paw to the stimulated whisker were recorded.

Rotarod motor test. In the rotarod motor test, only animals capable of remaining on the rotarod cylinder for $>300 \mathrm{~s}$ were used for experiments. The rotarod cylinder was accelerated from 0 to $10 \mathrm{rpm}$ within $1 \mathrm{~min}$, and the time that each animal remained on the rotarod was measured with a cutoff time of $300 \mathrm{~s}$. Data are presented as the mean duration from three trials.

B

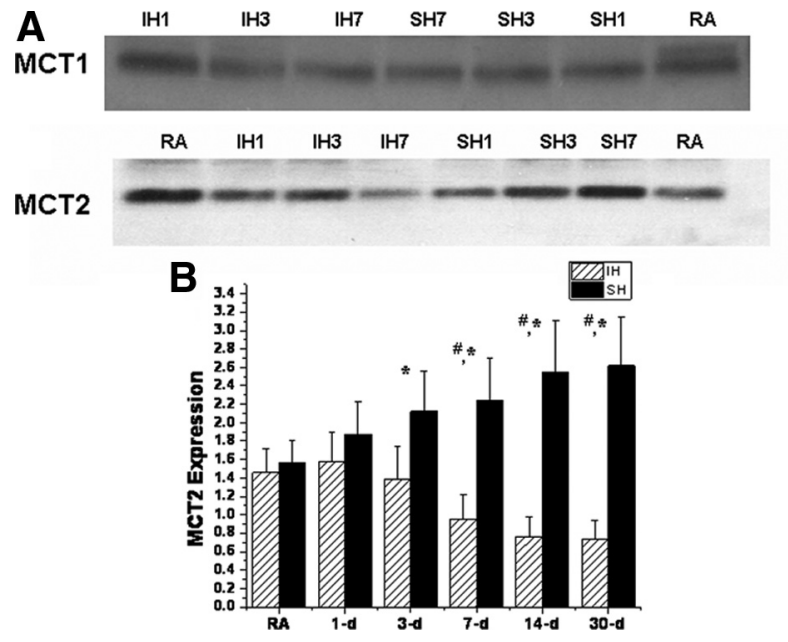

Figure 5. $A$, Representative immunoblots for MCT1 and MCT2 of cortical lysates after either IH or SH. $\boldsymbol{B}, \mathrm{MCT} 2$ protein expression changes over time in rat cortex after $\mathrm{IH}$ or $\mathrm{SH}$ exposures expressed as ratios against corresponding $\beta$-actin densitometric values $\left(n=6 /\right.$ group; ${ }^{*} p<$ 0.01 , SH vs RA; ${ }^{p} p<0.01$, IH vs RA).
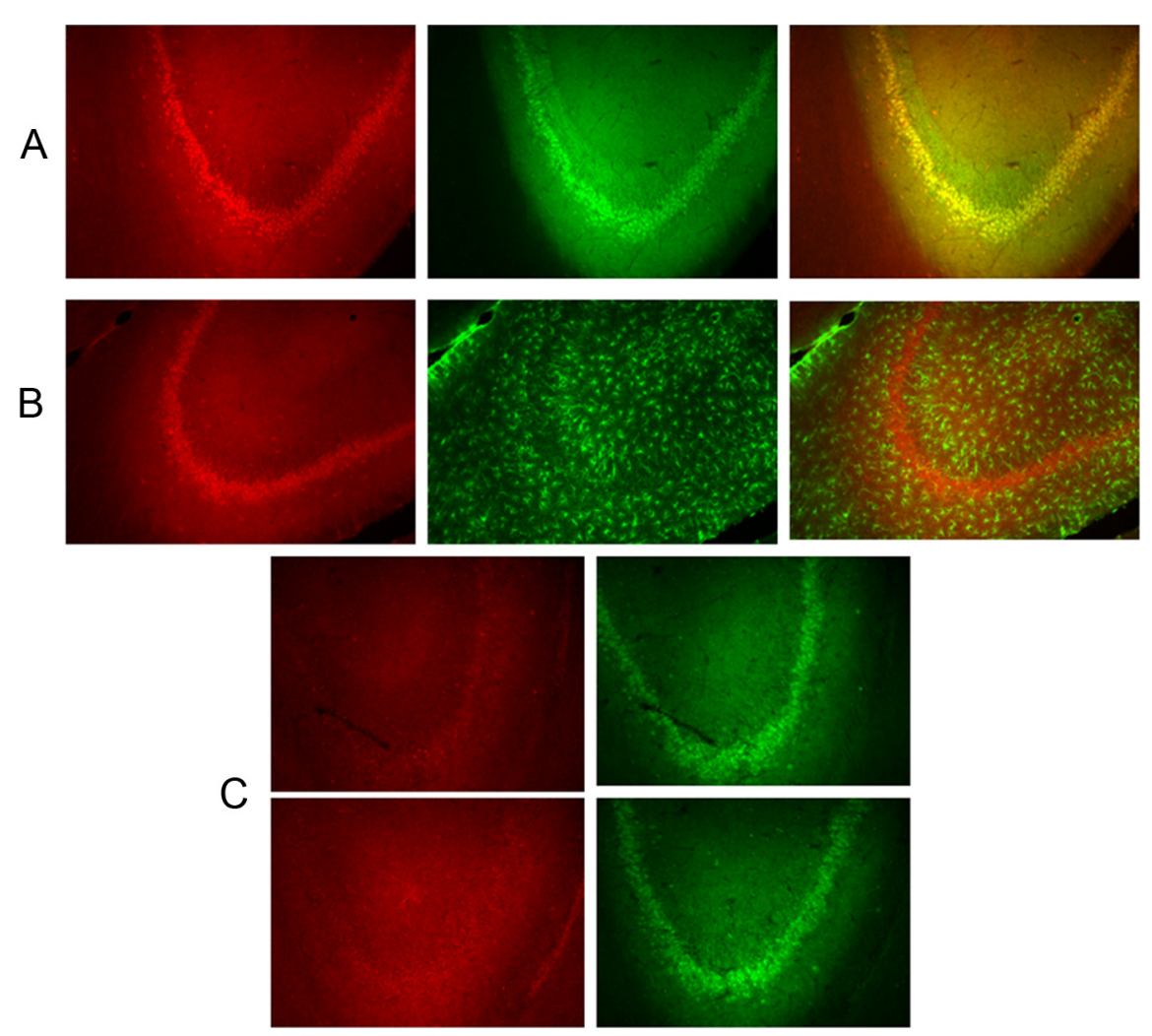

Figure 6. A, Immunohistochemical staining of MCT2 (red) and NeuN (green) and overlay in the hippocampus showing the high degree of colocalization of MCT2 in neurons. B, Immunohistochemical staining of MCT2 (red) and GFAP (green) and overlay in the hippocampus showing the relative absence of colocalization of MCT2 in glia. $C$, Immunohistochemical staining of MCT2 (red) and NeuN (green) in two different rats exposed to IH for $14 \mathrm{~d}$ showing marked reductions in the expression of MCT2.

Real-time PCR. Determinations of MCT1 mRNA and MCT2 mRNA were determined using real-time PCR procedures, Total RNA was extracted using RNeasy kit (Qiagen). Aliquots of total RNA ( $1 \mu \mathrm{g})$ were reverse transcribed to cDNA using random primers and Superscript II Reverse Transcriptase. cDNA equivalent to 100 ng of total RNA were subjected to real-time PCR analysis. The cycling conditions consisted of one cycle at $95^{\circ} \mathrm{C}$ for $10 \mathrm{~min}$ and 40 three-segment cycles $\left(95^{\circ} \mathrm{C}\right.$ for $15 \mathrm{~s}$, $59^{\circ} \mathrm{C}$ for $1 \mathrm{~min}$, and $72^{\circ} \mathrm{C}$ for $30 \mathrm{~s}$ ). 
Immunoblot analyses. Rats were exposed to $0,1,3,7,14$, or $30 \mathrm{~d}$ of $\mathrm{IH}$ or $\mathrm{SH}$ and were killed with a pentobarbital overdose. The skull was rapidly opened, and the brain was extracted, immediately placed on dry ice, and dissected under surgical microscopy. A portion of parietofrontal cortex and the CA1 region of the hippocampus were carefully removed. Tissues from two to three animals were pooled and homogenized at $0^{\circ} \mathrm{C}$ with a tissue blender in $20 \mathrm{~mm}$ Tris- $\mathrm{HCl}$ buffer, $\mathrm{pH} 7.5$, containing $2 \mathrm{~mm}$ EDTA, $0.5 \mathrm{~mm}$ EGTA, $25 \mu \mathrm{g} / \mathrm{ml}$ leupeptin, $25 \mu \mathrm{g} / \mathrm{ml}$ aprotinin, and $1 \mathrm{~mm}$ PMSF. The homogenate was centrifuged for $10 \mathrm{~min}$ at $1000 \times g$ at $4^{\circ} \mathrm{C}$ to remove cell debris. Protein content was measured in each soluble fraction using the Bradford method (DC Bio-Rad protein assay), and samples were frozen at $-70^{\circ} \mathrm{C}$ until analysis. Homogenate proteins $(50 \mu \mathrm{g})$ were heated for $10 \mathrm{~min}$ at $90^{\circ} \mathrm{C}$, loaded onto $8 \%$ PAGE gels, then transferred electrophoretically onto nitrocellulose membranes. Membranes were incubated overnight at $4^{\circ} \mathrm{C}$ with antibodies to MCT1 (1:2000) and MCT2 (1:2000) isoforms (Pierre et al., 2002; Pellerin et al., 2005), The same membranes were also blotted with $\beta$-actin antibody (Sigma), and blots were then normalized to $\beta$-actin. Densitometric analysis was performed with a gel scanning densitometer (Molecular Dynamics). In initial experiments, a control lysate was included.

Immunohistochemistry. Animals were deeply anesthetized and perfused intracardially with $4 \%$ phosphate-buffered paraformaldehyde. Serial sections were cut on a microtome. The free-floating sections were incubated with anti-MCT2 (1:200 dilution) and either anti-NeuN (1: $1000)$ or anti-GFAP antibodies (1:5000). Immunostained sections were further visualized with FITC-conjugated or rhodamine-conjugated second antibody. Sections were assessed using a Nikon Ellipse E800 microscope, and images were acquired using a SPOT digital camera.

Data analysis

Values are reported as mean \pm SD unless indicated otherwise. Two-way ANOVA for repeated measures, followed by the Newman-Keuls or Bonferroni's post hoc tests or Student's $t$ tests were used to compare across experimental conditions as appropriate. A $p$ value of $<0.05$ was considered to achieve statistical significance.

\section{Results}

IH, but not $\mathrm{SH}$, increases hippocampal slice vulnerability to acute severe hypoxia

No differences in ASH susceptibility emerged in $\mathrm{SH}$-exposed rats at 3, 7, or $14 \mathrm{~d}$ exposures when compared with normoxic rats (Fig. 1). In contrast, although no changes occurred after $3 \mathrm{~d}$ of $\mathrm{IH}$, slices harvested from rats exposed to either 7 or $14 \mathrm{~d}$ of $\mathrm{IH}$ were significantly more susceptible to ASH as evidenced by the proportion of slices showing reemergence of fEPSP within $30 \mathrm{~min}$ (Fig. 1) $(p<0.0001)$. Of note, lactate tissue levels were similar immediately after ASH in all conditions $(6.7 \pm 1.4 \mathrm{mM}$ after room air; $7.2 \pm 1.7 \mathrm{~mm}$ after $\mathrm{SH}$, and $6.8 \pm 1.6 \mathrm{~mm}$ after $\mathrm{IH} ; p>0.05)$.

\section{Infarct volume after MCAO and functional recovery in IH-exposed rats}

Based on the increased susceptibility to ASH after IH exposures, rats were exposed to $14 \mathrm{~d}$ of IH or normoxia (RA). Figure 2 shows infarct size after the MCAO procedure at 3 and $14 \mathrm{~d}$ of recovery. All IH-exposed rats had significantly larger infarcts compared with RA controls $(p<0.001)$. Indeed, mean infarct volume $3 \mathrm{~d}$ after MCAO was $318 \pm 43 \mathrm{~mm}^{3}$ in $\mathrm{IH}$-exposed rats $(n=12)$, whereas mean infarct volume was $223 \pm 38 \mathrm{~mm}^{3}$ in the control group (Fig. 2) $(n=12 ; p<0.001)$, and no infarct was present in sham-operated animals ( $n=6$ in $\mathrm{IH}$ and $n=4$ in normoxia) (data not shown). Although infarct size was smaller at $14 \mathrm{~d}$ of recovery, the extent of the infarct remained larger in $\mathrm{IH}$-exposed animals (Fig. 2). In addition, the overall performance on the various behavioral tasks was significantly worse in IH-exposed rats after either a 3, 7, or $14 \mathrm{~d}$ recovery period after MCAO when compared with RA-exposed rats (Fig. 3 ) ( $n=12$ /group). Indeed,

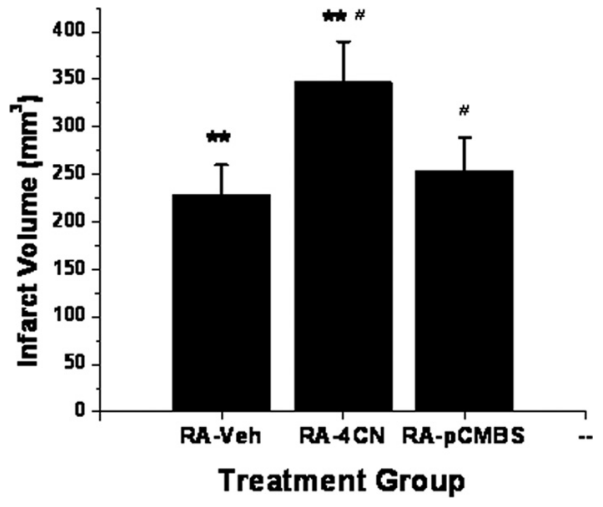

Figure 7. Infarct volumes in normoxic rats subjected to MCAO after intracerebroventricular treatment with MCT blockers 4-CN, pCMBS, or vehicle (Veh) $\left(^{* *} p<0.01,4-\mathrm{CN}\right.$ vs vehicle; ${ }^{\#} p<$ 0.01 , pCMBS vs $4-C N ; n=6$ /group).
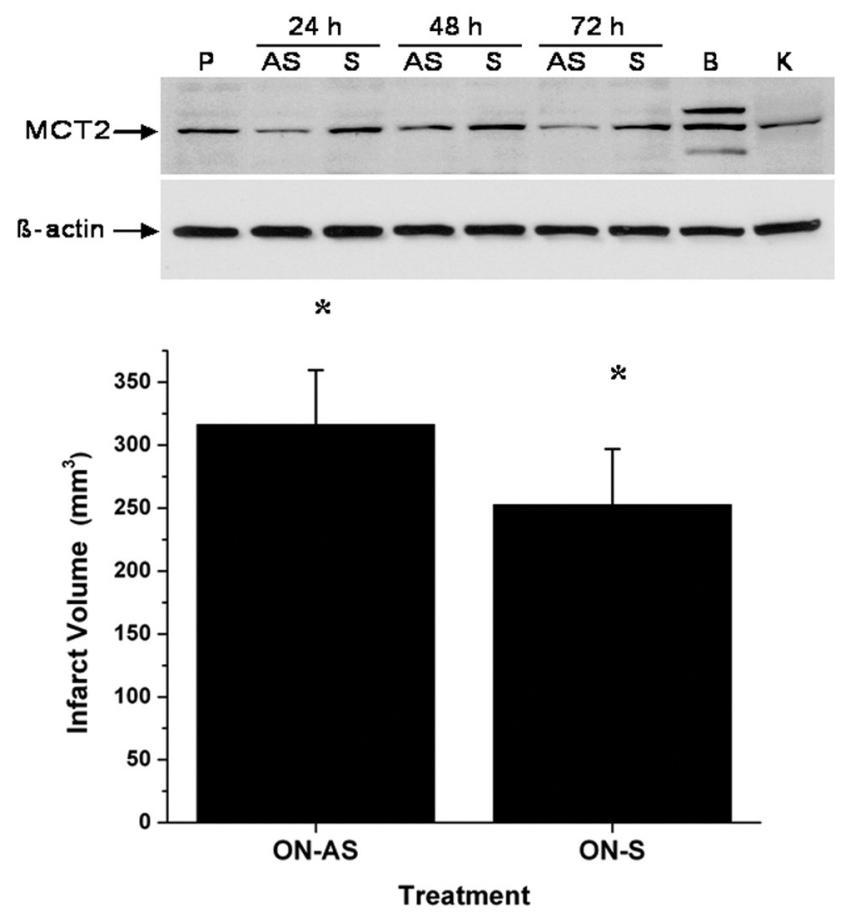

Figure 8. Top, Western blots of cortical lysates harvested from rats 24,48 , and $72 \mathrm{~h}$ after receiving $M C T 2$ antisense $0 \mathrm{~N}(A S)$ or scrambled ON (S), showing effective reductions in the expression of MCT2. P, Vehicle control; B, lysate from primary neuronal cell culture; $K$, control peptide. Bottom, Infarct volumes after MCAO in rats treated with either MCT2 antisense $0 \mathrm{~N}$ (AS) or scrambled $0 \mathrm{~N}(\mathrm{~S})\left({ }^{*} p<0.02 ; n=5 /\right.$ group $)$.

the overall time-dependent trajectory in the 18-point neurological scale was significantly worse in $\mathrm{IH}$-exposed animals during the acute phase (i.e., $3 \mathrm{~d}$ after MCAO and subsequent assessments) (Fig. 3A). Similar significant differences in the temporal changes in the right whisker to right paw responses occurred, albeit to a lesser extent (Fig. 3B). The overall number of foot misplacements was significantly higher (Fig. $3 C$ ), and the latency to fall in the rotarod task was significantly shorter in the IHexposed rats after MCAO and was followed by a slower recovery (Fig. 3D).

\section{MCT expression}

Overall MCT1 mRNA expression in cortex and hippocampus (data not shown) was unchanged by $\mathrm{SH}$ or $\mathrm{IH}$ over time. In 
contrast, increased expression of MCT2 occurred over time after $\mathrm{SH}$, whereas progressive reductions in MCT2 gene expression were apparent after $7 \mathrm{~d}$ of $\mathrm{IH}$ exposures when compared with normoxic timematched controls (Fig. 4).

Western blots of cortical lysates further confirmed the absence of any significant changes in MCT1 protein expression, whereas increases in MCT2 expression after long-term SH and reduced MCT2 expression after IH emerged (Fig. 5). Of note, MCT2 expression remained lower immediately 30-60 min after MCAO in IHexposed rats ( $n=3$ /group; $p<0.04)$.

Immunohistochemical analysis of hippocampal sections showed that MCT2 expression was preferentially circumscribed to neurons (NeuN-positive cells) (Fig. 6A) and that GFAP positively labeled cells did not exhibit intense staining (Fig. 6B). Furthermore, $\mathrm{IH}$ exposures for $14 \mathrm{~d}$ resulted in marked decreases in hippocampal expression of MCT2 in neurons (Fig. 6A, C).

\section{MCT pharmacological experiments and MCT2 anti-sense oligonucleotides and siRNA}

Normoxic rats implanted with 4-CN-containing osmotic pumps and subjected to MCAO showed significant increases in infarct size after $3 \mathrm{~d}$ when compared with rats receiving vehicle (Fig. 7) ( $n=8$ /group; $p<0.001)$. However, administration of the MCT1 blocker pCMBS, which does not affect the activity of MCT2 (Carpenter and Halestrap, 1994; Halestrap and Price, 1999; Manning Fox et al., 2000), failed to modify the size of the infarct after MCAO (Fig. 7) ( $n=6$ /group).

Similarly, MCT2 antisense ON administration resulted in anticipated reduced expression of MCT2 protein and increased infarct volumes after MCAO (Fig. 8) $(n=5 ; p<0.02)$. In addition, selective targeting of MCT2 using siRNAs strategies resulted in increased infarct size after MCAO (Fig. 9).

To examine whether exogenously administered pyruvate improved infarct size after MCAO, rats exposed to either IH or RA were administered intraperitoneal $500 \mathrm{mg} / \mathrm{kg}$ pyruvate or vehicle within $1 \mathrm{~h}$ from reperfusion after MCAO. Pyruvate treatment was associated with significant reductions in infarct size in RAexposed rats but failed to alter the extent of the infarct in $\mathrm{IH}$ exposed animals (Fig. 10).

\section{Infarct size in transgenic mice overexpressing hMCT2}

hMCT2 Tg mice and wild-type littermates were exposed to $14 \mathrm{~d}$ of IH or RA, after which they underwent MCAO procedures. Figure 10 shows that hMCT2 Tg mice were protected and that their infarct volumes in the cortex, striatum, or overall were smaller even after IH exposures when compared with wild-type mice (Fig. 11) ( $p<0.001 ; n=6 /$ group).

\section{Discussion}

The principal findings of this study include increased neuronal susceptibility to ASH in animals undergoing chronic exposures to intermittent hypoxia during sleep, mimicking the highly prevalent disorder of sleep apnea. Furthermore, we now show that this increased susceptibility is mediated, at least in part, by chronic IH-induced decreases in the neuronal expression of
B

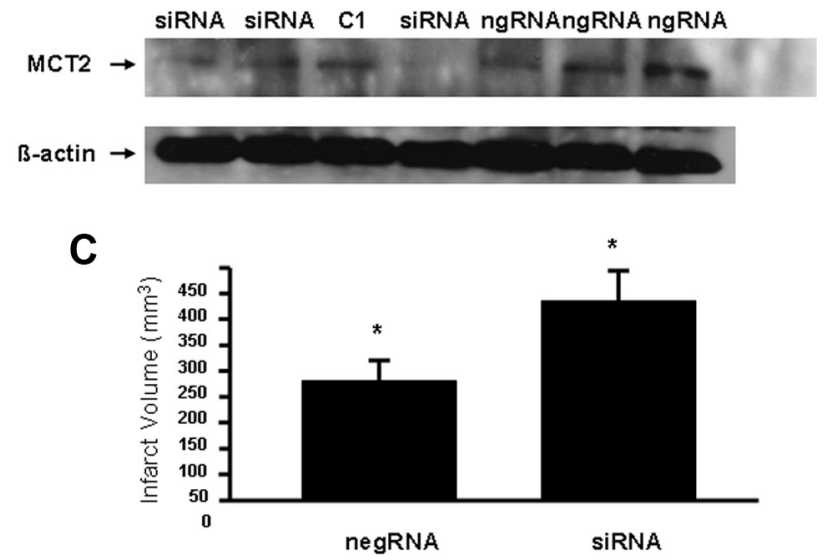

negRNA SiRNA

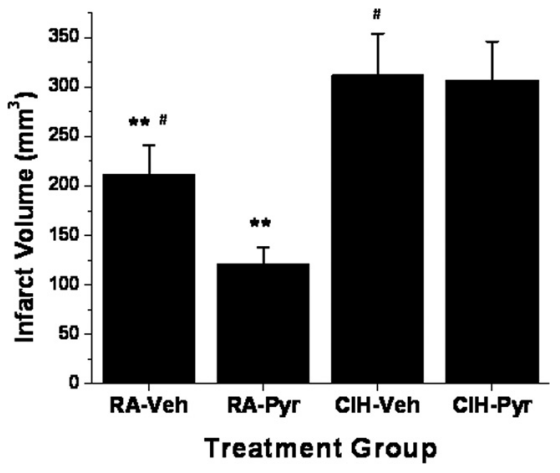

Figure 10. Infarct volumes in rats exposed to either $\mathrm{IH}$ for $14 \mathrm{~d}(\mathrm{CIH})$ or RA, and receiving intraperitoneal $500 \mathrm{mg} / \mathrm{kg}$ pyruvate (Pyr) or vehicle (Veh) within $1 \mathrm{~h}$ from reperfusion after MCA0 ${ }^{* *} p<0.01$, RA-Pyr vs RA-Veh, $n=6 /$ group; ${ }^{\#} p<0.02$, CIH-Pyr or CIH-Veh vs RA-Veh; $p$ value not significant, (IH-Pyr vs (IH-Veh, $n=6 /$ group).

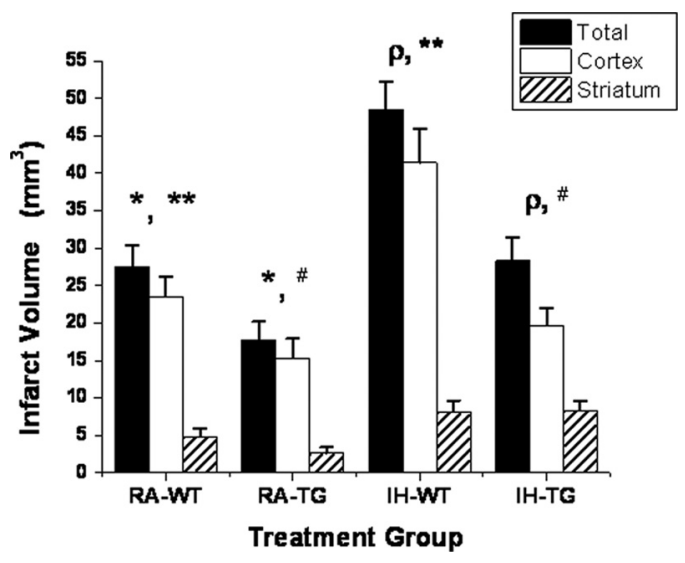

Figure 11. Infarct volumes in transgenic mice that overexpress human monocarboxylate trans porter 2 and their wild-type littermates subjected to MCAO after $14 \mathrm{~d}$ of IH exposures or normoxia. Significant reductions in infarct size emerged in normoxic hMCT2 Tg mice compared with wild-type (WT) littermates ( ${ }^{*} p<0.01, n=8 /$ group). However, IH exposures induced increases in infarct volume in wild-type mice $\left({ }^{* *} p<0.001\right.$ vs RA-WT) that were markedly attenuated in $\mathrm{hMCT} 2 \mathrm{Tg}$ mice $\left({ }^{\rho} p<0.01,{ }^{\#} p<0.01, n=8\right.$ /group; $p$ value not significant, IH-TG vs RA-WT). 
MCT2, such that alternative sources of energy to neurons during and after ASH cannot be effectively delivered. Together, these results present opportunities to further understand the mechanism underlying the dysregulation of MCT2 in the context of chronic IH and the development of effective strategies aiming at promoting MCT2 expression in patients with sleep apnea who may be at risk for cerebrovascular ischemic events (Minoguchi et al., 2007).

The conceptual framework of a monocarboxylate-dependent energy source alternative to neuronal function and survival has been the subject of substantial debate over the past 2 decades. Notwithstanding, the overall evidence would support the existence of a lactate shuttle between astrocytes and neurons that plays a critical role during conditions such as acute ischemiareperfusion injury (Schurr et al., 1988), as well as during physiological conditions such as learning and memory (Prichard et al., 1991; Allaman et al., 2011; Suzuki et al., 2011). As part of this shuttle, MCT2 has emerged as a critical constituent of neuronal bioenergetic pathways. MCT2 cannot only be expressed in the plasma membrane, in which it governs the transport of monocarboxylates into the cell, but is also expressed in mitochondria, in which it is associated with lactate dehydrogenase and cytochrome oxidase, thereby further supporting the existence of a neuronal mitochondrial monocarboxylate oxidation complex shuttle and that of a cell-cell-based monocarboxylate shuttle, similar to those identified previously in other non-CNS tissues (Brooks, 2002; Hashimoto et al., 2008). The neuronal monocarboxylate oxidation complex described recently by Brooks and colleagues would thus allow neurons to increase cell monocarboxylate functional availability and to oxidize available monocarboxylates, regardless of their sources (i.e., glycolytic pathways in the same neuron, apposing astrocytes, or from the microvasculature). Indeed, Moreira et al. (2009) have shown recently that both MCT1 and MCT2 are markedly upregulated in microglia, suggesting that these cells may also play a role in the recovery process from ischemic stroke. Accordingly, and consistent with previous findings (Pellerin and Magistretti, 1994), such observations would permit operation of both shuttles in brain, thereby accounting for their critical role in both physiological and pathological conditions.

Despite such obvious implications, it remains essentially unknown, however, how MCTs are regulated under conditions such as hypoxia/ischemia, in which enhanced lactate metabolism is required for survival (McClelland and Brooks, 2002; Py et al., 2005). Preliminary evidence would suggest that the presence of hypoxia would enhance the expression of MCTs, and indeed at least one of the genes coding for MCTs, namely MCT4, was upregulated by hypoxia at the transcriptional level via a hypoxiainducible factor 1-dependent mechanism (Ullah et al., 2006). We have recently shown that the transcription of the MCT2 gene is initiated from five distinct variants of exon 1, likely under the control of five alternative promoters, and that a large number of mature mRNA are produced from these pre-mRNA through alternative splicing of multiple internal exons in the $5^{\prime}$-UTR region. These findings would suggest that both alternative promoter usage and alternative splicing are key elements in the cellular- and subcellular-specific expression patterns of MCT2, as well as in the gene expression responses to environmental stressors (Zhang et al., 2007).

From a functional standpoint, we found discrepant changes in hippocampal slice vulnerability to acute ischemia only after animals had been exposed to IH, and yet exposures to a similar magnitude of hypoxia in which the stimulus was sustained was void of any adverse consequences after acute ischemia. Similarly, the structural and functional outcomes of MCAO were adversely affected by preceding IH. Such findings would suggest differential regulation of MCT expression in $\mathrm{IH}$ and $\mathrm{SH}$, despite the fact that the magnitude of hypoxia was the same. Confirmation of such assumption emerged from the reported expression studies, whereby IH and SH clearly had discrepant effects on MCT2 expression levels in brain, with significant reduction in MCT2 at the mRNA and protein levels after IH. Of note, the expression patterns of MCT2 in control animals were analogous to those reported previously (Koehler-Stec et al., 1998; Pierre et al., 2002). We would also emphasize that the differences in infarct size were confirmed in wild-type mice exposed to $\mathrm{IH}$ and were attenuated by overexpression of MCT2 in mice even after exposures to $\mathrm{IH}$.

The use of pyruvate or lactate as alternative energy sources during ASH has been repeatedly advocated as potentially conferring substantial neuronal protection in various model of stroke. Indeed, Lee et al. (2001) showed that exogenous administration of pyruvate at doses similar to those used herein was associated with substantial neuroprotection in a forebrain stroke model in rats. Similar findings have been reported recently by Alano et al. (2010), whereby bypassing glycolytic inhibition associated with ASH with pyruvate prevented mitochondrial failure and neuronal cell death. In our model, pyruvate was effective in affording neuroprotection in normoxic animals but did not confer any incremental benefit in IH-exposed rats. Such observations would be commensurate with the reduction in MCT2 expression, which would then restrict the ability to activate the putative neuronal mitochondrial monocarboxylate oxidation complex and that of a cell-cell-based monocarboxylate shuttle to enable improved availability of cellular ATP. It remains unclear, however, whether such mechanisms would also be able to favorably regulate mitochondrial oxidative stress in the context of ASH (Fiskum et al., 2004). Such assumption may not be far-fetched considering the life-prolonging effect of increased mitochondrial pyruvate availability in a Caenorhabditis elegans model (Mouchiroud et al., 2011).

In summary, prolonged exposures to the intermittent hypoxia during sleep, which characterizes the highly prevalent condition of sleep apnea, result in increased brain susceptibility to acute ischemic events aiming to reproduce stroke in a rodent. The enhanced vulnerability of the neural substrate in this context appears to be accounted for by concomitant reductions in MCT2 neuronal expression. Thus, efforts to promote expression and efficiency of neuronal monocarboxylate transporters may ameliorate the adverse outcomes of stroke in patients with underlying sleep apnea.

\section{References}

Alano CC, Garnier P, Ying W, Higashi Y, Kauppinen TM, Swanson RA (2010) $\mathrm{NAD}^{+}$depletion is necessary and sufficient for poly(ADP-ribose) polymerase-1-mediated neuronal death. J Neurosci 30:2967-2978.

Ali LK, Avidan AY (2008) Sleep-disordered breathing and stroke. Rev Neurol Dis 5:191-198.

Allaman I, Bélanger M, Magistretti PJ (2011) Astrocyte-neuron metabolic relationships: for better and for worse. Trends Neurosci 34:76-87.

Bassetti C, Aldrich MS (1999) Sleep apnea in acute cerebrovascular diseases: final report on 128 patients. Sleep 22:217-223.

Beebe DW, Groesz L, Wells C, Nichols A, McGee K (2003) The neuropsychological effects of obstructive sleep apnea: a meta-analysis of normreferenced and case-controlled data. Sleep 26:298-307.

Berthet C, Lei H, Thevenet J, Gruetter R, Magistretti PJ, Hirt L (2009) Neuroprotective role of lactate after cerebral ischemia. J Cereb Blood Flow Metab 29:1780-1789. 
Brooks GA (2002) Lactate shuttles in nature. Biochem Soc Trans 30: $258-264$.

Carpenter L, Halestrap AP (1994) The kinetics, substrate and inhibitor specificity of the lactate transporter of Ehrlich-Lettre tumour cells studied with the intracellular pH indicator BCECF. Biochem J 304:751-760.

Colle LM, Holmes LJ, Pappius HM (1986) Correlation between behavioral status and cerebral glucose utilization in rats following freezing lesion. Brain Res 397:27-36.

Fellows LK, Boutelle MG, Fillenz M (1993) Physiological stimulation increases nonoxidative glucose metabolism in the brain of the freely moving rat. J Neurochem 60:1258-1263.

Fiskum G, Rosenthal RE, Vereczki V, Martin E, Hoffman GE, Chinopoulos C, Kowaltowski A (2004) Protection against ischemic brain injury by inhibition of mitochondrial oxidative stress. J Bioenerg Biomembr 36:347-352.

Garcia JH, Wagner S, Liu KF, Hu XJ (1995) Neurological deficit and extent of neuronal necrosis attributable to middle cerebral artery occlusion in rats. Statistical validation. Stroke 26:627-634; discussion 635.

Good DC, Henkle JQ, Gelber D, Welsh J, Verhulst S (1996) Sleep-disordered breathing and poor functional outcome after stroke. Stroke 27:252-259.

Gozal D, Daniel JM, Dohanich GP (2001) Behavioral and anatomical correlates of chronic episodic hypoxia during sleep in the rat. J Neurosci 21:2442-2450.

Halestrap AP, Price NT (1999) The proton-linked monocarboxylate transporter (MCT) family: structure, function and regulation. Biochem J 343:281-299.

Hashimoto T, Hussien R, Cho HS, Kaufer D, Brooks GA (2008) Evidence for the mitochondrial lactate oxidation complex in rat neurons: demonstration of an essential component of brain lactate shuttles. PLoS One 3:e2915.

Kheirandish L, Gozal D (2006) Neurocognitive dysfunction in children with sleep disorders. Dev Sci 9:388-399.

Koehler-Stec EM, Simpson IA, Vannucci SJ, Landschulz KT, Landschulz WH (1998) Monocarboxylate transporter expression in mouse brain. Am J Physiol 275:E516-E524.

Kreisman NR, Soliman S, Gozal D (2000) Regional differences in hypoxic depolarization and swelling in hippocampal slices. J Neurophysiol 83: 1031-1038.

Lee JY, Kim YH, Koh JY (2001) Protection by pyruvate against transient forebrain ischemia in rats. J Neurosci 21:RC171(1-6).

Manning Fox JE, Meredith D, Halestrap AP (2000) Characterisation of human monocarboxylate transporter 4 substantiates its role in lactic acid efflux from skeletal muscle. J Physiol 529:285-293.

McClelland GB, Brooks GA (2002) Changes in MCT 1, MCT 4, and LDH expression are tissue specific in rats after long-term hypobaric hypoxia. J Appl Physiol 92:1573-1584.

Minoguchi K, Yokoe T, Tazaki T, Minoguchi H, Oda N, Tanaka A, Yamamoto M, Ohta S, O’Donnell CP, Adachi M (2007) Silent brain infarction and platelet activation in obstructive sleep apnea. Am J Respir Crit Care Med 175:612-617.

Moreira TJ, Pierre K, Maekawa F, Repond C, Cebere A, Liljequist S, Pellerin L (2009) Enhanced cerebral expression of MCT1 and MCT2 in a rat ischemia model occurs in activated microglial cells. J Cereb Blood Flow Metab 29:1273-1283.

Mouchiroud L, Molin L, Kasturi P, Triba MN, Dumas ME, Wilson MC, Halestrap AP, Roussel D, Masse I, Dallière N, Ségalat L, Billaud M, Solari F (2011) Pyruvate imbalance mediates metabolic reprogramming and mimics lifespan extension by dietary restriction in Caenorhabditis elegans. Aging Cell 10:39-54.

Nowak M, Kornhuber J, Meyrer R (2006) Daytime impairment and neurodegeneration in OSAS. Sleep 29:1521-1530.

Parra O, Arboix A, Bechich S, García-Eroles L, Montserrat JM, López JA, Ballester E, Guerra JM, Sopeña JJ (2000) Time course of sleep-related breathing disorders in first-ever stroke or transient ischemic attack. Am J Respir Crit Care Med 161:375-380.

Pellerin L, Magistretti PJ (1994) Glutamate uptake into astrocytes stimulates aerobic glycolysis: a mechanism coupling neuronal activity to glucose utilization. Proc Natl Acad Sci U S A 91:10625-10629.

Pellerin L, Bergersen LH, Halestrap AP, Pierre K (2005) Cellular and subcellular distribution of monocarboxylate transporters in cultured brain cells and in the adult brain. J Neurosci Res 79:55-64.
Pierre K, Magistretti PJ, Pellerin L (2002) MCT2 is a major neuronal monocarboxylate transporter in the adult mouse brain. J Cereb Blood Flow Metab 22:586-595.

Prichard J, Rothman D, Novotny E, Petroff O, Kuwabara T, Avison M, Howseman A, Hanstock C, Shulman R (1991) Lactate rise detected by $1 \mathrm{H}$ NMR in human visual cortex during physiologic stimulation. Proc Natl Acad Sci U S A 88:5829-5831.

Py G, Eydoux N, Lambert K, Chapot R, Koulmann N, Sanchez H, Bahi L, Peinnequin A, Mercier J, Bigard AX (2005) Role of hypoxia-induced anorexia and right ventricular hypertrophy on lactate transport and MCT expression in rat muscle. Metabolism 54:634-644.

Ratan RR, Siddiq A, Smirnova N, Karpisheva K, Haskew-Layton R, McConoughey S, Langley B, Estevez A, Huerta PT, Volpe B, Roy S, Sen CK, Gazaryan I, Cho S, Fink M, LaManna J (2007) Harnessing hypoxic adaptation to prevent, treat, and repair stroke. J Mol Med 85:1331-1338.

Ryan CM, Bayley M, Green R, Murray BJ, Bradley TD (2011) Influence of continuous positive airway pressure on outcomes of rehabilitation in stroke patients with obstructive sleep apnea. Stroke 42:1062-1067.

Sahlin C, Sandberg O, Gustafson Y, Bucht G, Carlberg B, Stenlund H, Franklin KA (2008) Obstructive sleep apnea is a risk factor for death in patients with stroke: a 10-year follow-up. Arch Intern Med 168:297-301.

Schallert T, Woodlee MT, Fleming SM (2003) Experimental focal ischemic injury: behavior-brain interactions and issues of animal handling and housing. ILAR J 44:130-143.

Schurr A, Rigor BM (1998) Brain anaerobic lactate production: a suicide note or a survival kit? Dev Neurosci 20:348-357.

Schurr A, West CA, Rigor BM (1988) Lactate-supported synaptic function in the rat hippocampal slice preparation. Science 240:1326-1328.

Schurr A, Payne RS, Miller JJ, Rigor BM (1997a) Brain lactate is an obligatory aerobic energy substrate for functional recovery after hypoxia: further in vitro validation. J Neurochem 69:423-426.

Schurr A, Payne RS, Miller JJ, Rigor BM (1997b) Brain lactate, not glucose, fuels the recovery of synaptic function from hypoxia upon reoxygenation: an in vitro study. Brain Res 744:105-111.

Schurr A, Payne RS, Tseng MT, Gozal E, Gozal D (2001) Excitotoxic preconditioning elicited by both glutamate and hypoxia and abolished by lactate transport inhibition in rat hippocampal slices. Neurosci Lett 307:151-154.

Somers VK, White DP, Amin R, Abraham WT, Costa F, Culebras A, Daniels S, Floras JS, Hunt CE, Olson LJ, Pickering TG, Russell R, Woo M, Young T; American Heart Association Council for High Blood Pressure Research Professional Education Committee, Council on Clinical Cardiology; American Heart Association Stroke Council; American Heart Association Council on Cardiovascular Nursing; American College of Cardiology Foundation (2008) Sleep apnea and cardiovascular disease: an American Heart Association/American College Of Cardiology Foundation Scientific Statement from the American Heart Association Council for High Blood Pressure Research Professional Education Committee, Council on Clinical Cardiology, Stroke Council, and Council On Cardiovascular Nursing. In collaboration with the National Heart, Lung, and Blood Institute National Center on Sleep Disorders Research (National Institutes of Health). Circulation 118:1080-1111.

Spriggs DA, French JM, Murdy JM, Curless RH, Bates D, James OF (1992) Snoring increases the risk of stroke and adversely affects prognosis. Q J Med 83:555-562.

Suzuki A, Stern SA, Bozdagi O, Huntley GW, Walker RH, Magistretti PJ, Alberini CM (2011) Astrocyte-neuron lactate transport is required for long-term memory formation. Cell 144:810-823.

Swanson RA, Morton MT, Tsao-Wu G, Savalos RA, Davidson C, Sharp FR (1990) A semiautomated method for measuring brain infarct volume. J Cereb Blood Flow Metab 10:290-293.

Ullah MS, Davies AJ, Halestrap AP (2006) The plasma membrane lactate transporter MCT4, but not MCT1, is up-regulated by hypoxia through a HIF-1alpha-dependent mechanism. J Biol Chem 281:9030-9037.

Valham F, Mooe T, Rabben T, Stenlund H, Wiklund U, Franklin KA (2008) Increased risk of stroke in patients with coronary artery disease and sleep apnea: a 10-year follow-up. Circulation 118:955-960.

Zhang SX, Searcy TR, Wu Y, Gozal D, Wang Y (2007) Alternative promoter usage and alternative splicing contribute to mRNA heterogeneity of mouse monocarboxylate transporter 2. Physiol Genomics 32:95-104. 